\title{
O perfil da produção científica neuropsicológica no Brasil: uma revisão integrativa
}

The profile of neuropsychological scientific production in Brazil: an integrative review

El perfil de la producción científica neuropsicológica en Brasil: una revisión integrativa

Leila Karla Barboza Legnani ${ }^{1}$, Tiago Pereira de Souza ${ }^{2}$

${ }^{1}$ Psicóloga. Pós-graduanda em Neuropsicologia. Centro Universitário Amparense UNIFIA.

Amparo, São Paulo

${ }^{2}$ Doutorando em Educação em Ciências pela Universidade Federal do Rio Grande do Sul. Docente do Centro Universitário Amparense UNIFIA. Amparo, São Paulo

\section{RESUMO}

\begin{abstract}
A neuropsicologia surgiu a partir de interações entre diferentes áreas e busca compreender a relação cérebro e mente, com enfoque na base anatômica das funções cognitivas. O presente artigo apresenta um panorama da neuropsicologia no Brasil nos últimos cinco anos, por meio de uma revisão integrativa. Os dados foram coletados selecionando cinco recursos informacionais: Literatura Latino Americano e do Caribe em Ciências da Saúde - LILACS, Scientific Electronic Library Online - SciELO, Periódicos Eletrônicos em Psicologia - PePSIC, Index Psi Periódicos Técnico-Científicos e Google acadêmico. A amostra final é composta por 20 artigos, cuja
\end{abstract}

Autor de Correspondência:

*Tiago Pereira de Souza. E-mail: tiago.ps.fono@gmail.com 
abordagem metodológica predominante é do tipo quantitativa. O periódico com mais publicações foi a Revista Neuropsicologia Latinoamericana e, as palavras-chave identificadas, "neuropsicologia", "cognição" e "funções executivas" foram as mais adotadas nos artigos. Concluiu-se, com a presente revisão bibliográfica integrativa, que a temática mais abordada no contexto da neuropsicologia no Brasil são os transtornos mentais.

Palavras-chave: Neuropsicologia. Disfunção Cognitiva. Cognição.

\begin{abstract}
Neuropsychology arose from interactions among different areas and seeks to understand the brain and mind relationship, focusing the anatomical basis of cognitive functions. This article presents an overview of neuropsychology in Brazil in the last five years, through an integrative review. Data were collected by selecting five information resources: Latin American and Caribbean Literature in Health Sciences - LILACS, Scientific Electronic Library Online SciELO, Electronic Journals in Psychology - PePSIC, Index Psi Technical-Scientific Journals, and Google scholar. The final sample consists of 20 articles, whose predominant methodological approach is quantitative. The journal with most publications was Revista Neuropsicologia Latinoamericana and the keywords identified, "neuropsychology", "cognition", and "executive functions", were the most adopted in the articles. It was concluded, with the present integrative bibliographic review, that the most discussed topic in the context of neuropsychology in Brazil is mental disorders.
\end{abstract}

Keywords: Neuropsychology. Cognitive Dysfunction. Cognition.

\title{
RESUMEN
}

La neuropsicología surgió a partir de interacciones entre diferentes áreas y busca comprender la relación cerebro-mente, con un enfoque en la base anatómica de las funciones cognitivas. Este artículo presenta un panorama de la neuropsicología en Brasil en los últimos cinco años, a través de una revisión integradora. Los datos fueron recolectados mediante la selección de cinco recursos de información: Literatura Latinoamericana y del Caribe en Ciencias de la Salud LILACS, Biblioteca Electrónica Científica en Línea - SciELO, Revistas Electrónicas de Psicología - PePSIC, Revistas Técnico-Científicas Index Psi y Google académico. La muestra final está formada por 20 artículos, cuyo enfoque metodológico predominante es de tipo cuantitativo. El periódico con más publicaciones fue la Revista Neuropsicología Latinoamericana y las palabras clave identificadas, "neuropsicología", "cognición" y "funciones ejecutivas" fueron las más adoptadas en los artículos. Se concluyó, con la presente revisión bibliográfica.

Palabras clave: Neuropsicología. Disfunción Cognitiva. Cognición. 


\section{INTRODUÇÃO}

A Neuropsicologia é uma área do conhecimento que busca estudar o cérebro e sua organização complexa, ou seja, visa analisar a relação existente entre fatores cognitivos, comportamentais e ações do sistema nervoso, identificando sujeitos com patologias. A neuropsicologia relaciona-se à medicina, principalmente com a neurologia, com caráter multi, inter e transdisciplinar. Esta área do conhecimento é resultante de outras áreas fundamentais (tais como neuroanatomia, neurofisiologia, neuroquímica e neurofarmacologia) e aplicadas (tais como psicometria, psicologia clínica e experimental, psicopatologia e psicologia cognitiva). Ainda, baseia-se em matérias cujo enfoque está no estudo do funcionamento do cérebro e da conduta humana e as consequências dessa interação, visando entender como cérebro e mente originam a implexa realidade humana ${ }^{1,2}$

A principal ferramenta prática desta ciência é a Avaliação Neuropsicológica (AN), aplicada nas diferentes faixas etárias com desenvolvimento normal ou patológico. Para a AN é preciso analisar o comportamento do indivíduo em contextos variados (em família, em sociedade ou no ambiente escolar quando aplicável $)^{3,4}$. Para sua aplicação, utiliza-se de "entrevistas, observações, provas de rastreio e testes psicométricos para identificar rendimento cognitivo funcional e investigar a integridade ou comprometimento de uma determinada função cognitiva"1. Ademais, fornece subsídios para diagnosticar clinicamente, entender o perfil cognitivo, estabelecer prognósticos bem como definir programas de reabilitação e a mensuração da responsividade do paciente ao tratamento ${ }^{1,3,4}$.

No Brasil, tem-se observado o desenvolvimento da neuropsicologia, no que diz respeito, por exemplo, à quantidade de profissionais especializados, bem como de publicações acerca do tema. Em função da interdisciplinariedade das áreas de atuação, pesquisas e intervenções neuropsicológicas vêm promovendo debates sobre a alcance da área, de maneira especial no que diz respeito ao uso e progresso das ferramentas para avaliação neuropsicológica ${ }^{5}$.

$\mathrm{Na}$ atualidade, a formação em Neuropsicologia clínica ocorre por meio de cursos de pós-graduação lato sensu, ou seja, através de especializações reconhecidas pelo Ministério da Educação. Ainda, existem pósgraduações cujas linhas de orientação direcionam para esta temática, em nível de especialização, mestrado e/ou doutorado 5 .

Frente à interdisciplinaridade da área, é necessário desenvolver estudos sobre os temas relacionados à neuropsicologia, principalmente no que tange à promoção de pesquisas e intervenções neuropsicológicas, as quais colaboram para a difusão dos debates. Ademais, mapear a produção científica sobre determinada área do conhecimento fornece subsídios para aprofundar conhecimentos sobre aquilo que vem sendo abordado e a forma de aplicação dos conhecimentos teóricos.

Este estudo apresenta ao leitor uma síntese sobre as produções científicas no período de 2015 a 2020, demostrando um panorama de pesquisas no que diz respeito a 3 grupos de estudos: transtornos mentais, funções cognitivas e ensino.

\section{MÉTODO}

Configura-se uma revisão integrativa de literatura, capaz de analisar resultados de forma sistemática, ordenada, abrangente, produzindo uma síntese da construção do conhecimento do tema em sua integralidade ${ }^{6}$.

A sistematização da pesquisa foi ordenada por seis fases metodológicas: a) formulação do problema ou questão de pesquisa; b) estabelecimento de critérios para a coleta de dados na literatura; c) definição das informações a serem extraídas do material 
pesquisado; d) avaliação e categorização dos estudos selecionados; e) análise e interpretação dos dados obtidos na busca; f) apresentação da síntese do conhecimento proposto com a revisão integrativa ${ }^{7}$.

A concepção da questão a ser respondida por esta revisão, utilizou o recurso PECO (População, Exposição, Controle e Desfecho) $)^{8}$, possibilitando determinar os aspectos reguladores na elaboração da pesquisa. A "população" estudada foram os pesquisadores da área da neuropsicologia, a "exposição" está vinculada à produção científica publicada no período de 2015 à 2020, o "controle" não se aplica a este estudo, uma vez que não foram feitas comparações entre os artigos publicados, encontrados e classificados no "desfecho". Em seguida, delimitou-se a questão que norteia a presente pesquisa: "Qual o perfil da produção científica neuropsicológica brasileira?”.
A análise dos estudos pré-selecionados, descritos na Figura 01, foi feita por dois pesquisadores (L.K.B.L. e T.P.S.) de maneira autônoma, de modo que, na impraticabilidade de acordo (situação não evidenciada no processo) em qualquer uma das etapas, um terceiro revisor seria consultado, evitando assim, vieses na seleção dos estudos. Inicialmente, os títulos e resumos foram analisados a partir de critérios predeterminados para a etapa de rastreamento, envolvendo a compatibilidade temática com a neuropsicologia. Na última etapa, a leitura na íntegra dos artigos, levou em consideração, como critério de elegibilidade, a necessidade de apresentação de resultados inéditos, afim de responder à questão desta revisão integrativa, eliminando artigos de revisão de literatura. Os estudos encontrados foram importados para o gerenciador de referências Zotero.
Figura 01 - Fluxograma do Processo de Seleção $0^{7,9}$.

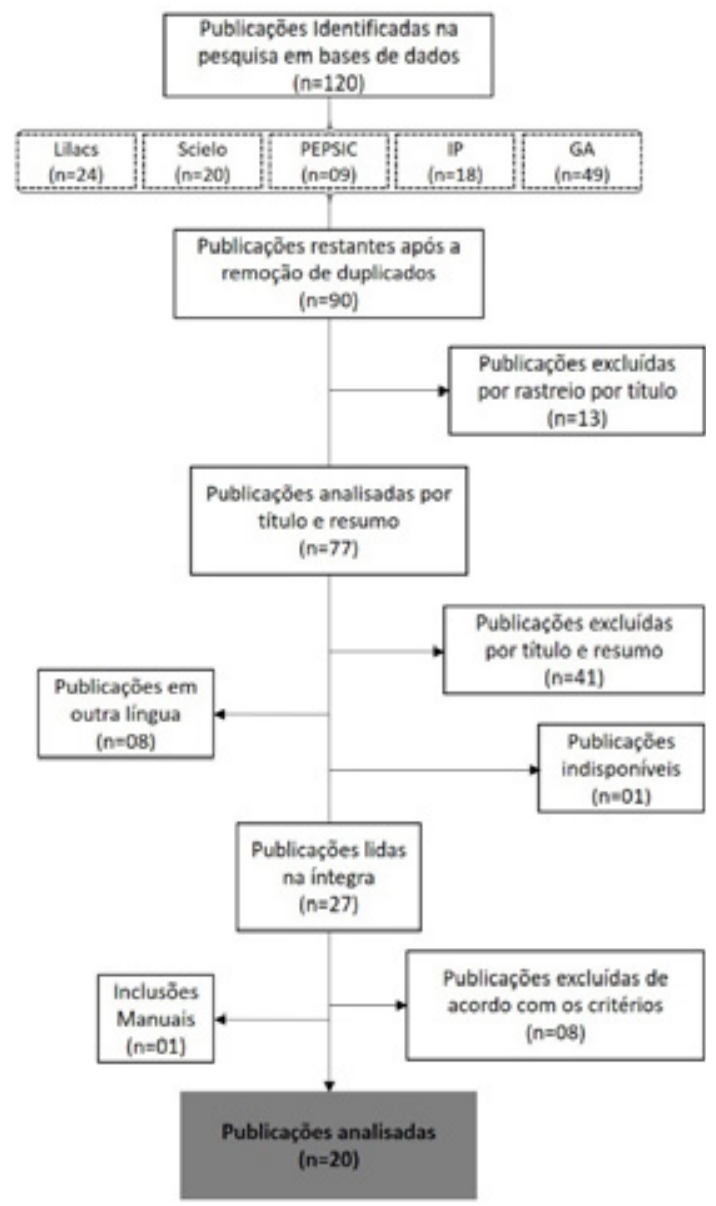

Fonte: Autores. 
$\mathrm{Na}$ fase de seleção, aplicou-se os descritores "neuropsicologia" or "neuropsicológico", na busca em títulos, resumos e assuntos. Os dados foram coletados utilizando a selecionando cinco recursos informacionais: base de dados eletrônica da área da saúde (Literatura Latino Americano e do Caribe em Ciências da Saúde - LILACS), biblioteca digital (Scientific Electronic Library Online - SciELO), portal de Periódicos Eletrônicos de Psicologia (PePSIC), Index Psi Periódicos Técnico-Científicos e um buscador acadêmico (Google acadêmico).

Além das bases de dados indexadas abrangeuse a literatura cinzenta, e a complementação do levantamento dos estudos ocorreu por meio de busca manual nas citações e referências dos artigos primários identificados. Como critérios de inclusão, considerou-se: ser artigo original, possuir texto completo, estar publicado no período de 2015 a agosto de 2020.

A fase de extração dos dados foi organizada por meio de um instrumento específico para este fim, registrado em planilha eletrônica de forma independente pelos pesquisadores (L.K.B.L. e T.P.S) e, posteriormente comparada, de modo que, na existência de discrepâncias (situação não evidenciada), um terceiro revisor seria consultado. O processo permitiu a identificação do título, periódico, método, palavras-chave e classificação por categorias. Os trabalhos selecionados foram, então, sumarizados e categorizados de acordo com os seguintes critérios: funções cognitivas, ensino da neuropsicologia e transtornos mentais.

\section{RESULTADOS}

A partir da busca foram obtidos 120 estudos e, então, realizou-se a leitura dos títulos e resumos, levando em consideração os critérios de inclusão e exclusão, dos quais foram selecionados 20 estudos para análise. A fim de facilitar a identificação dos estudos selecionados, estes foram organizados em um quadro (Quadro 1), conforme apresentado a seguir:

Quadro 1 - Artigos selecionados

\begin{tabular}{|c|c|c|c|c|}
\hline Título & Periódico & Método & Palavras-Chave & Categoria \\
\hline $\begin{array}{l}\text { Atenção, memória e percepção: } \\
\text { uma análise conceitual da } \\
\text { Neuropsicologia aplicada à } \\
\text { propaganda e sua influência no } \\
\text { comportamento do consumidor }{ }^{10}\end{array}$ & $\begin{array}{l}\text { Revista Brasileira } \\
\text { de Ciências da } \\
\text { Comunicação }\end{array}$ & Qualitativo & $\begin{array}{l}\text { Atenção. Memória. Percepção. } \\
\text { Neuropsicologia. Propaganda. }\end{array}$ & $\begin{array}{l}\text { Funções } \\
\text { cognitivas }\end{array}$ \\
\hline $\begin{array}{l}\text { Perfil neuropsicológico de } \\
\text { pacientes com amputação maior de } \\
\text { membros inferiores por etiologia } \\
\text { vascular }^{11}\end{array}$ & Acta fisiátrica & Quantitativo & $\begin{array}{l}\text { Neuropsicologia. Cognição. } \\
\text { Testes Psicológicos. Doença } \\
\text { Arterial Periférica. Amputação. }\end{array}$ & $\begin{array}{l}\text { Funções } \\
\text { cognitivas }\end{array}$ \\
\hline $\begin{array}{l}\text { Perfil neuropsicológico pós- } \\
\text { traumatismo cranioencefálico: } \\
\text { há sempre extensas sequelas } \\
\text { cognitivas? }{ }^{12}\end{array}$ & $\begin{array}{l}\text { Ciências \& } \\
\text { Cognição }\end{array}$ & $\begin{array}{l}\text { Estudo de } \\
\text { Caso }\end{array}$ & $\begin{array}{c}\text { Traumatismo cranioencefálico. } \\
\text { Avaliação neuropsicológica. } \\
\text { Funções executivas. } \\
\text { Neurocognição. }\end{array}$ & $\begin{array}{l}\text { Funções } \\
\text { cognitivas }\end{array}$ \\
\hline $\begin{array}{l}\text { Desfecho neurológico, } \\
\text { neuropsicológico e } \\
\text { neuropsiquiátrico de um caso de } \\
\text { cavernoma mesencefálico pós- } \\
\text { cirúrgico }^{13}\end{array}$ & $\begin{array}{l}\text { Revista } \\
\text { Neuropsicologia } \\
\text { Latinoamericana }\end{array}$ & $\begin{array}{l}\text { Estudo de } \\
\text { Caso }\end{array}$ & $\begin{array}{l}\text { Cavernoma mesencéfalo. } \\
\text { Memória episódica verbal. } \\
\text { Funções executivas. } \\
\text { Neurocirurgia. }\end{array}$ & $\begin{array}{l}\text { Funções } \\
\text { cognitivas }\end{array}$ \\
\hline
\end{tabular}




\begin{tabular}{|c|c|c|c|c|}
\hline $\begin{array}{l}\text { Avaliação neuropsicológica no } \\
\text { acidente vascular cerebral: um } \\
\text { estudo de caso }^{14}\end{array}$ & Distúrb. Comun & $\begin{array}{l}\text { Estudo de } \\
\text { Caso }\end{array}$ & $\begin{array}{l}\text { Neuropsicologia. Acidente } \\
\text { Vascular Cerebral. Afasia. }\end{array}$ & $\begin{array}{l}\text { Funções } \\
\text { cognitivas }\end{array}$ \\
\hline $\begin{array}{l}\text { O “Querer Ir Para Casa” no } \\
\text { Alzheimer: traços de identidade } \\
\text { e subjetividade em uma narrativa } \\
\text { emergente durante a aplicação de } \\
\text { um teste neuropsicológico }{ }^{15}\end{array}$ & $\begin{array}{c}\text { Revista } \\
\text { Intercâmbio }\end{array}$ & Qualitativo & $\begin{array}{l}\text { Alzheimer. Identidade. } \\
\text { Subjetividade. Narrativa. } \\
\text { Interação. }\end{array}$ & $\begin{array}{l}\text { Transtorno } \\
\text { mental }\end{array}$ \\
\hline $\begin{array}{l}\text { Perfil neuropsicológico típico e } \\
\text { atípico na demência de Alzheimer: } \\
\text { dificuldades diagnósticas em três } \\
\text { estudos de caso }^{16}\end{array}$ & $\begin{array}{l}\text { Polêmica } \\
\text { - Revista } \\
\text { Eletrônica da } \\
\text { UERJ }\end{array}$ & $\begin{array}{l}\text { Estudo de } \\
\text { Caso }\end{array}$ & $\begin{array}{c}\text { Doença de Alzheimer. } \\
\text { Neuropsicologia. Demência. } \\
\text { Diagnóstico. }\end{array}$ & $\begin{array}{l}\text { Transtorno } \\
\text { mental }\end{array}$ \\
\hline $\begin{array}{l}\text { Reabilitação neuropsicológica e } \\
\text { terapia cognitivo-comportamental } \\
\text { aplicadas a paciente com doença de } \\
\text { Alzheimer }{ }^{17}\end{array}$ & $\begin{array}{l}\text { Revista Brasileira } \\
\text { de Terapia } \\
\text { Comportamental } \\
\text { e Cognitiva }\end{array}$ & $\begin{array}{l}\text { Estudo de } \\
\text { Caso }\end{array}$ & $\begin{array}{c}\text { Doença de Alzheimer. } \\
\text { Reabilitação neuropsicológica. } \\
\text { Terapia cognitivo- } \\
\text { comportamental. Idoso. }\end{array}$ & $\begin{array}{l}\text { Transtorno } \\
\text { mental }\end{array}$ \\
\hline $\begin{array}{l}\text { Diagnóstico neuropsicológico } \\
\text { diferencial entre o Transtorno } \\
\text { Depressivo Maior e o } \\
\text { Comprometimento Cognitivo Leve: } \\
\text { estudo de caso }^{18}\end{array}$ & $\begin{array}{l}\text { Revista } \\
\text { Neuropsicologia } \\
\text { Latinoamericana }\end{array}$ & $\begin{array}{l}\text { Estudo de } \\
\text { Caso }\end{array}$ & $\begin{array}{l}\text { Avaliação neuropsicológica. } \\
\text { Transtorno Depressivo Maior. } \\
\text { Comprometimento Cognitivo } \\
\text { Leve. Funções executivas. } \\
\text { Memória episódica. }\end{array}$ & $\begin{array}{l}\text { Transtorno } \\
\text { mental }\end{array}$ \\
\hline $\begin{array}{l}\text { Breve perfil peuropsicológico dos } \\
\text { usuários de crack do estado de } \\
\text { Rondônia }^{19}\end{array}$ & $\begin{array}{l}\text { Revista } \\
\text { Científica da } \\
\text { FAEMA }\end{array}$ & Qualitativo & $\begin{array}{l}\text { Mini exame do estado mental. } \\
\text { Avaliação do estado mental. } \\
\text { Usuários de crack. }\end{array}$ & $\begin{array}{l}\text { Transtorno } \\
\text { mental }\end{array}$ \\
\hline $\begin{array}{l}\text { Estudo exploratório do ensino da } \\
\text { neuropsicologia nos currículos } \\
\text { dos cursos de graduação em } \\
\text { fonoaudiologia }^{20}\end{array}$ & CoDAS & Quantitativo & $\begin{array}{l}\text { Fonoaudiologia. } \\
\text { Neuropsicologia. Educação } \\
\text { Superior. Neurociência } \\
\text { Cognitiva. Neurologia. }\end{array}$ & Ensino \\
\hline $\begin{array}{l}\text { A neuropsicologia e o psicodrama } \\
\text { com ênfase no Teste de } \\
\text { Espontaneidade }^{21}\end{array}$ & $\begin{array}{c}\text { Revista eletrônica } \\
\text { de Trabalhos } \\
\text { Acadêmicos }\end{array}$ & $\begin{array}{l}\text { Quali- } \\
\text { quantitativo }\end{array}$ & $\begin{array}{l}\text { Neuropsicologia. Psicodrama. } \\
\text { Teste de espontaneidade. }\end{array}$ & Ensino \\
\hline $\begin{array}{l}\text { A prática da avaliação psicológica } \\
\text { em neuropsicologia e psicologia } \\
\text { do trânsito durante estágio de } \\
\text { formação profissional }^{22}\end{array}$ & $\begin{array}{l}\text { Revista } \\
\text { Psicologia e } \\
\text { Saúde }\end{array}$ & Qualitativo & $\begin{array}{l}\text { Avaliação psicológica. Prática. } \\
\text { Pesquisa. Neuropsicologia. } \\
\text { Psicologia do trânsito. }\end{array}$ & Ensino \\
\hline $\begin{array}{l}\text { Avaliação das estratégias de leitura } \\
\text { em leitores típicos e disléxicos: } \\
\text { abordagem neuropsicológica }^{23}\end{array}$ & $\begin{array}{c}\text { Revista } \\
\text { Psicologia: } \\
\text { Teoria e Prática }\end{array}$ & Quantitativo & $\begin{array}{l}\text { Cognição. Avaliação. } \\
\text { Linguagem. Transtorno de } \\
\text { aprendizagem. Leitura. }\end{array}$ & $\begin{array}{l}\text { Transtorno } \\
\text { mental }\end{array}$ \\
\hline $\begin{array}{l}\text { Efeito de idade e escolaridade } \\
\text { no instrumento de avaliação } \\
\text { neuropsicológica breve } \\
\text { NEUPSILIN }^{24}\end{array}$ & Psico-USF & Quantitativo & $\begin{array}{l}\text { Cognição. Envelhecimento. } \\
\text { Educação. Neuropsicologia. }\end{array}$ & $\begin{array}{l}\text { Funções } \\
\text { cognitivas }\end{array}$ \\
\hline $\begin{array}{l}\text { Evidências de validade do } \\
\text { Subteste Aritmética do TDE- } \\
\text { II: da Psicometria Moderna à } \\
\text { Neuropsicologia Cognitiva }^{25}\end{array}$ & $\begin{array}{c}\text { Revista } \\
\text { Neuropsicologia } \\
\text { Latinoamericana }\end{array}$ & Quantitativo & $\begin{array}{l}\text { Teste de Desempenho Escolar. } \\
\text { Aritmética, Teoria de Resposta } \\
\text { ao Item. Neuropsicologia } \\
\text { Cognitiva. Funções Executivas. }\end{array}$ & $\begin{array}{l}\text { Transtorno } \\
\text { mental }\end{array}$ \\
\hline $\begin{array}{l}\text { Modelo Neuropsicológico- } \\
\text { Fuzzy para subsidiar a análise de } \\
\text { procedimentos educacionais em } \\
\text { Engenharia de Produção }{ }^{26}\end{array}$ & $\begin{array}{c}\text { Revista de Ensino } \\
\text { de Engenharia }\end{array}$ & Quantitativo & $\begin{array}{c}\text { Funções cognitivas. Redes } \\
\text { Neuro-Fuzzy. Neuropsicologia, } \\
\text { Fuzzy. }\end{array}$ & $\begin{array}{l}\text { Funções } \\
\text { cognitivas }\end{array}$ \\
\hline
\end{tabular}




\begin{tabular}{|c|c|c|c|c|}
\hline $\begin{array}{l}\text { Neupsilin-Inf em um modelo de } \\
\text { avaliação neuropsicológica breve } \\
\text { para centros de saúde }{ }^{27}\end{array}$ & Psico-USF & Quantitativo & $\begin{array}{l}\text { Avaliação breve. } \\
\text { Neuropsicologia. } \\
\text { Desenvolvimento. Cognição. } \\
\text { Saúde pública. }\end{array}$ & $\begin{array}{c}\text { Transtorno } \\
\text { mental }\end{array}$ \\
\hline $\begin{array}{l}\text { Perfil neuropsicológico em crianças } \\
\text { com perturbação de hiperatividade } \\
\text { com déficit de atenção: avaliação da } \\
\text { memória de trabalho }^{28}\end{array}$ & $\begin{array}{c}\text { Acta Pediátrica } \\
\text { Portuguesa }\end{array}$ & Quantitativo & $\begin{array}{l}\text { Criança. Memória de Curto } \\
\text { Prazo. Testes de Linguagem. } \\
\text { Testes Neuropsicológicos. } \\
\text { Transtorno do Défice de } \\
\text { Atenção com Hiperatividade. } \\
\text { Transtornos de Aprendizagem. }\end{array}$ & $\begin{array}{c}\text { Transtorno } \\
\text { mental }\end{array}$ \\
\hline $\begin{array}{l}\text { Triagem infantil neuropsicológica } \\
\text { computadorizada para indicação do } \\
\text { TDAH em crianças e adolescentes }{ }^{29}\end{array}$ & $\begin{array}{l}\text { Psicologia } \\
\text { Argumento }\end{array}$ & Quantitativo & $\begin{array}{l}\text { Neuropsicologia. Avaliação } \\
\text { Neuropsicológica Infantil. } \\
\text { Testagem Neuropsicológica } \\
\text { Computadorizada. TDAH. }\end{array}$ & $\begin{array}{c}\text { Transtorno } \\
\text { mental }\end{array}$ \\
\hline
\end{tabular}

Fonte: Os autores.

Ao analisar o Quadro, constata-se que a predominância metodológica é do tipo quantitativa (45\%), estudo de caso (30\%), qualitativa (20\%) e do tipo quali-quantitativo $(5 \%)$. No que diz respeito aos periódicos de publicação, destacam-se Revista Neuropsicologia Latinoamericana $(n=3)$ e Psico-USF $(n=2)$, os outros estudos foram publicados em revistas diferentes.

As palavras-chave apresentadas com maior frequência foram: neuropsicologia $(n=11)$; cognição $(n=4)$; funções executivas $(n=4)$; Alzheimer $(n=3)$; avaliação neuropsicológica $(n=3)$ e TDAH $(n=2)$, o restante dos termos foram apontados apenas uma vez.

A classificação proposta foi feita considerando o objetivo, resultados e conclusões dos estudos, os quais foram divididos em três grupos: Funções cognitivas, Transtorno mental e Ensino. Com relação à classificação por categoria analisada, transtorno mental representa $50 \%$ da amostra selecionada, seguida por funções cognitivas e ensino, 35\% e 15\% respectivamente.

$\mathrm{Na}$ categoria "Transtorno mental” foram incluídos dez artigos ${ }^{15-19,23,25,27-29}$, nos quais a neuropsicologia e/ou avaliação neuropsicológica está em um contexto específico de estudo de algum transtorno mental descrito no DSM-5 ${ }^{30}$. Na categoria funções cognitivas, foram incluídos sete $\operatorname{artigos}^{10-14,24,26}$ e, nestes, a proposta principal era avaliar as funções cognitivas em contextos variados, sem relacionar com um transtorno mental especifico. Por fim, na categoria "Ensino", cuja proposta era abordar a neuropsicologia nas áreas de ensino, foram incluídos $3 \operatorname{artigos}^{20-22}$.

Pode-se observar, também, um perfil das publicações, no qual a predominância metodológica é do tipo quantitativa, seguida por estudo de caso, qualitativa e quali-quantitativo, respectivamente. O periódico com maior número de publicações é a Revista Neuropsicologia Latinoamericana e, no que tange às palavras-chave identificadas, neuropsicologia, cognição e funções executivas foram as mais adotadas nos artigos.

\section{DISCUSSÃO}

\section{Transtornos Mentais}

Uma das principais finalidades da AN é identificar pessoas com lesão cerebral, diferenciando-as dos sujeitos normais e, ainda, encontrar prejuízos estruturais suspeitos resultantes de padrões de comportamento numa prova constituída por avaliações variadas ${ }^{31}$, tal como constatado nos 
$\operatorname{artigos}^{16,18,19,23,25,27-29}$.

Estabelecer diagnósticos confiáveis sobre transtornos mentais é fundamental para definir tratamentos adequados, determinar índices de prevalência para que serviços de saúde mental sejam planejados bem como selecionar grupamentos para desenvolvimento de pesquisas e documentar dados ${ }^{30}$, conforme apresentado pelos estudos ${ }^{16-18,25,27,29}$.

Neste cenário, como indicam os estudos ${ }^{16,17,19}$, a avaliação diagnóstica de transtornos mentais deve considerar as experiências, os sintomas e os comportamentos do paciente, pois estes sofrem variações conforme o contexto sociocultural no qual estão inseridos, bem como influencia no processo de adaptação ao tratamento ${ }^{30,32}$.

Além disso, os transtornos neuropsicológicos comumente abrangem distintos graus de comprometimento de funções como memória, atenção, linguagem, funções executivas, habilidades perceptivo-motoras, dentre outras, presentes em quadros de distúrbios neurológicos e/ou neuropsiquiátricos, do desenvolvimento ou adquiridos $^{32}$. Estas variações foram constatadas nos estudos ${ }^{18,19,23,25,27,28}$, nos quais foram verificados prejuízos cognitivos no transtorno depressivo maior, nos usuários de crack, transtornos do neurodesenvolvimento, em pacientes disléxicos, pessoas com TDAH e em pacientes com Alzheirmer.

A dislexia e o TDAH, patologias apontadas em alguns $\operatorname{artigos}^{23,28,29}$, são condições clínicas, entre outras, classificadas no DSM-5 ${ }^{30}$, como transtornos do neurodesenvolvimento. Esses transtornos envolvem restrições características no aprendizado ou nas funções cognitivas, resultando em prejuízos nas inter-relações sociais, prejudicando o desempenho escolar dos indivíduos, que carecem de adequações para seu processo de aprendizagem visando alcançar seus potenciais ${ }^{30,33}$.

O transtorno depressivo maior (TDM), segundo o DSM-5 $5^{30}$, compreende a classe dos transtornos disruptivos da regulação do humor e, neste transtorno especifico (TDM) um dos critérios diagnósticos é a capacidade diminuída de pensar ou se concentrar, conforme evidenciado no $\operatorname{artigo~}^{18}$. Já o Alzheimer, apresentado nos estudos ${ }^{15-1} 7$, é um dos transtornos neurocognitivos, conforme classificação do DSM-5 $5^{30}$, com prejuízo em um ou mais domínios cognitivos.

\section{Funções Cognitivas}

As funções cognitivas, apresentadas nos artigos ${ }^{10-14,24}$, são explicadas como "constelações de sistemas funcionais complexos que se inter-relacionam com constelações de estruturas cerebrais que lhe servem de substrato"34, podendo ser classificadas como percepção, atenção, memória, linguagem e funções executivas, sendo a integração destas funções responsável por grande parte dos comportamentos humanos ${ }^{33,35}$.

Dentre os objetivos da AN tem-se o estabelecimento da existência ou não de disfunção cognitiva ainda em estágios iniciais ${ }^{34,36,37}$. Para tal, existem diferentes formas de avaliação cognitiva disponíveis e conhecidas e, inclusive, de baixo custo, tal qual apresentadas nos artigos ${ }^{11-14,24,26}$. Porém, sua eficácia varia em função de fatores como duração da aplicação e características do indivíduo ${ }^{34}$.

\section{Ensino da Neuropsicologia}

A neuropsicologia enquanto ciência interdisciplinar ${ }^{32}$, permite a aplicação e uso de seus conceitos nas diversas áreas do conhecimento. A integração entre instrumentais, metodologias e referenciais teóricos de diferentes áreas, como, por exemplo, Psicologia, Neurologia, Psiquiatria, Linguística, Psicolinguística, Neurolinguística, Fonoaudiologia, Farmacologia, Fisioterapia, Terapia Ocupacional, etc ${ }^{38}$, permite a introdução de temáticas da abordagem neuropsicológicas, já no processo de graduação dos profissionais. Os estudos ${ }^{20-22}$ 
correlacionaram a aplicabilidade, aprimoramento e presença de tais referências na formação de psicólogos e fonoaudiólogos.

\section{CONCLUSÕES}

O presente artigo teve por objetivo apresentar um panorama da produção científica neuropsicológica no Brasil nos últimos cinco anos. Foram selecionados 20 artigos, os quais foram agrupados em 3 categorias, sendo elas ensino, transtorno mental e funções cognitivas, das quais houve predominância dos artigos classificados como transtorno mental (50\% da amostra).

Assim, conclui-se, a partir da análise feita, que instituir diagnósticos fidedignos sobre transtornos mentais é essencial para deliberar sobre tratamentos apropriados, bem como para proposição de índices de prevalência visando o planejamento de serviços de saúde mental e estabelecimento de grupos para condução de pesquisas.

Por fim, é importante ressaltar a necessidade de novos estudos envolvendo neuropsicologia e o contexto brasileiro, seja a partir de estudos de casos, ou novas pesquisas bibliográficas exploratórias.

\section{REFERÊNCIAS}

1. Ramos AA, Hamdan AC. O crescimento da avaliação neuropsicológica no Brasil: uma revisão sistemática. Psicol Cienc Prof. 2016;36(2):471-85.

2. Jarros RB. Perfil neuropsicológico de adolescentes com transtornos de ansiedade [dissertação]. Porto Alegre: Universidade Federal do Rio Grande do Sul; 2011.

3. Carvalho AM, Guerra LB. Avaliação neuropsicológica na educação. In: Malloy-Diniz LF, Fuentes D, Mattos P, Abreu N. Avaliação neuropsicológica. Porto Alegre: Artmed; 2010. p. 327-28.
4. Corrêa PV. O estado da arte da neuropsicologia em diálogo com a escolarização: das limitações às possibilidades [dissertação]. Porto Velho: Fundação Universidade Federal de Rondônia; 2014.

5. Hazin I, Fernandes I, Gomes E, Garcia D. Neuropsicologia no Brasil: passado, presente e futuro. Estud Pesqui Psicol. 2018;18:1137-54.

6. Ercole FF, Melo LS, Alcoforado CLGC. Revisão integrativa versus revisão sistemática. REME. 2014;18(1):912.

7. Whittemore R, Knafl K. The integrative review: updated methodology. J Adv Nurs. 2005;52(5):546-53.

8. Moher D, Shamseer L, Clarke M, Ghersi D, Liberati A, Petticrew M, et al. Preferred reporting items for systematic review and meta-analysis protocols (PRISMA-P) 2015 statement. Syst Rev. 2015;4:1-9.

9. Mendes KDS, Silveira RCCP, Galvão CM. Revisão integrativa: método de pesquisa para a incorporação de evidências na saúde e na enfermagem. Texto Context Enferm. 2008;17(4):758-64.

10. Endo ACB, Roque MAB. Atenção, memória e percepção: uma análise conceitual da Neuropsicologia aplicada à propaganda e sua influência no comportamento do consumidor. Intercom Rev Bras Cienc Comum. 2017;40(1):77-96.

11. Pessoa CV, Paik S, Correia GP, Nobre IDN, Pardini ACG, Ingham SJM. Perfil neuropsicológico de pacientes com amputação maior de membros inferiores por etiologia vascular. Acta Fisiatr [Internet]. 2018 [citado em 13 ago 2020];25(3):131-7. Disponível em: https://www.revistas.usp. br/actafisiatrica/article/view/162674

12. Cotrena C, Figueiredo ÂL, Fonseca RP. Perfil neuropsicológico pós-traumatismo cranioencefálico: há sempre extensas sequelas cognitivas? Cienc Cogn. 2015;20(1):110-22

13. Zimmermann N, Pontes MC, Correa DG, Delaere FJ, Gasparetto EL, Faveret EC, et al. Desfecho neurológico, neuropsicológico e neuropsiquiátrico de um caso de cavernoma mesencefálico pós-cirúrgico. Neuropsi Latinoam [Internet]. 2016 [citado em 13 ago 2020];8(3):1121. Disponível em: https://www.neuropsicolatina.org/index. php/Neuropsicologia_Latinoamericana/article/view/314 
14. Pavan LS, Casarin FS, Pagliarin KC, Fonseca RP. Avaliação neuropsicológica no acidente vascular cerebral: um estudo de caso. Disturb Comun [Internet]. 2015 [citado em 13 ago 2020];27(4):831-9. Disponível em: http://revistas. pucsp.br/index.php/dic/article/view/22665/18815

15. Fronza SA, Cruz FM. O "querer ir para casa" no Alzheimer: traços de identidade e subjetividade em uma narrativa emergente durante a aplicação de um teste neuropsicológico. Intercâmbio [Internet]. 2020 [citado em 13 ago 2020];44:108-26. Disponível em: http://200.144.145.24/ intercambio/article/view/42814

16. Pinho MM, Norte CE, Chaves DNG, Charchat-Fichman H. Perfil neuropsicológico típico e atípico na demência de Alzheimer: dificuldades diagnósticas em três estudos de caso. Polêmica. 2018;18(3):129-39.

17. Dainez ECL. Reabilitação neuropsicológica e terapia cognitivo-comportamental aplicadas a paciente com doença de Alzheimer. Rev Bras Ter Comp Cogn [Internet]. 2017[citado em 13 ago 2020];19(1):146-54. Disponível em: www.usp.br/rbtcc/index.php/RBTCC/article/view/957/504

18. Goulart M, Tarrasconi M, Holz MR, Kochhann R. Diagnóstico neuropsicológico diferencial entre o Transtorno Depressivo Maior e o Comprometimento Cognitivo Leve: estudo de caso. Neuropsi Latinoam. 2019;11(2):34-45.

19. Rocha VHC, Lemos Setúbal CR, Calheiros PRV, Bergamini GB. Breve perfil neuropsicológico dos usuários de crack do estado de Rondônia. Rev Cient Fac Educ Meio Ambient [Internet]. 2018 [citado em 13 ago 2020];9(1):289300. Disponível em: http://www.faema.edu.br/revistas/ index.php/Revista-FAEMA/article/view/561

20. Brasil AC, Lara TL, Chaves MLF, Lawlor B, Beber BC. Estudo exploratório do ensino da neuropsicologia nos currículos dos cursos de graduação em fonoaudiologia. Codas. 2020;32(2):e20190023.

21. Albernaz MF. A neuropsicologia e o psicodrama com ênfase no teste de espontaneidade. Rev Electron Trab Acad [Internet]. 2017 [citado em 13 ago 2020];3:1-22. Disponível em: http://revista.universo.edu.br/index.php?journal=3GOIANIA4\&page $=$ article\&op=view\&path\%5B\%5D=3986

22. Santana JJRA, Soares MC. A prática da avaliação psicológica em neuropsicologia e psicologia do trânsito durante estágio de formação profissional. Rev Psicol Saude. 2019;11(1):141-54.
23. Dias NM, Minervino CASM, Reppold CT, Macedo EC, Seabra AG. Avaliação das estratégias de leitura em leitores típicos e disléxicos: abordagem neuropsicológica. Psicol Teoria Prat. 2015;17(2):171-84.

24. Rodrigues JC, Muller JL, Esteves C, Fonseca RP, Parente MAMP, Salles JF, et al. Efeito de idade e escolaridade no Instrumento de Avaliação Neuropsicológica Breve NEUPSILIN. Psico USF. 2018;23(2):319-32.

25. Viapiana VF, Giacomoni CH, Stein LM, Fonseca RP. Evidências de validade do Subteste Aritmética do TDEII: da psicometria moderna à neuropsicologia cognitiva. Neuropsi Latinoam. 2016;8(2):16-26.

26. Sucena MP, Silva DCC, Faria AF, Brito LS. Modelo Neuropsicológico-Fuzzy para subsidiar a análise de procedimentos educacionais em Engenharia de Produção. Rev Ensino Engen [Internet]. 2018 [citado em 13 ago 2020];37(2):122-33. Disponível em: http://revista.educacao. ws/revista/index.php/abenge/article/view/1133

27. Miranda MC, Sinnes EG, Zanini GAV, Baumgartner B, Salles JF, Fonseca RP, et al. Neupsilin-Inf em um Modelo de Avaliação Neuropsicológica Breve para Centros de Saúde. Psico USF. 2018;23(1):95-108.

28. Guardiano M, Candeias L, Guimarães JE, Viana V, Almeida P. Perfil neuropsicológico em crianças com perturbação de hiperatividade com déficit de atenção: avaliação da memória de trabalho. 2017 [citado em 13 ago 2020];48:229-35. Disponível em: https://repositorio-aberto. up.pt/handle/10216/110120

29. Popi JM, Riechi TIJS, Hamdan AC. Triagem infantil neuropsicológica computadorizada para indicação do TDAH em crianças e adolescentes. Psicol Argument. 2016;34(84):39-50.

30. American Psychiatric Association. DSM-5: Manual Diagnóstico e Estatístico de Transtornos Mentais. Porto Alegre: Artmed; 2014.

31. Simões MR. Utilizações da WISC-III na avaliação neuropsicológica de crianças e adolescentes. Paideia. 2002;12(23):113-32.

32. Haase VG, Salles JF, Miranda MC, Malloy-Diniz L, Abreu N, Argollo N, et al. Neuropsicologia como ciência interdisciplinar: consenso da comunidade brasileira de pesquisadores/clínicos em Neuropsicologia. Neuropsi Latinoam. 2012;4(4):1-8. 
33. Machado MS, Teixeira AR, Costa SS. Correlation between cognitive functions and central auditory processing in adolescents with non-cholesteatomatous chronic otitis media. Dement Neuropsychol. 2018;12(3):314-20.

34. Barbosa LMG. Introdução histórica sobre a relação entre mente e cérebro: da filosofia à neuropsicologia. São Paulo: Uninove; 2018.

35. Fonseca V. Papel das funções cognitivas, conativas e executivas na aprendizagem: uma abordagem neuropsicopedagógica. Rev Psicoped. 2014;31(96):236-53.

36. Mäder MJ. Avaliação neuropsicológica: aspectos históricos e situação atual. Psicol Cienc Prof. 1996;16(3):128.

37. Pessoa RMP. Transtornos Neurocognitivos: conceitos, comparação entre critérios diagnósticos e acurácia de testes neuropsicológicos em uma amostra de idosos da comunidade [dissertação]. Ribeirão Preto: Universidade de São Paulo; 2019.

38. Leis HR. Sobre o conceito de interdisciplinaridade. Cad Pesqui Interdiscip Cienc Human. 2005;6(73):2-23. 